\title{
Scanning Electron Microscopy
}

\section{Keiichi Tanaka ${ }^{1}$ and Kazuo Maeda ${ }^{2}$}

${ }^{1}$ Department of Anatomy (Emeritus), Tottori University Medical School, Tanaka SEM Institute, Yonago, Japan

${ }^{2}$ Department.of Obstetrics and Gynecology (Emeritus), Tottori University Medical School, Yonago, Japan

\section{Abstract}

Scanning electron microscope is an electron microscope, which electronically enlargesthe subject surface, where interesting images are illustrated in the natural history, biology and medicine.

\section{Introduction, Methods and Materials}

There areseveral microscopes, e.g. optical microscope, electron microscope, ultrasound microscope, where theauthor $\mathrm{T}$ dealt with scanning electron microscope, which differs from the transparentelectron microscope in the function. Transparent electron microscope needs very thin sample which is imaged by electron transparency in vacuum field. Scanning electron microscope (SEM) needs no transparent sample, but the surface of dry subject is scanned by secondary electron accelerated by the voltage between anode to cathode,which is usually 1.0 to $25 \mathrm{kV}$. The reflected electron is processed to display enlarged surface images of the subject for 10 to 800,000 folds [1-3], where actual size is shown by a scale barson the enlarged photos. The magnification is shown by “ $x$ ”, e.g. $\times 100$ is 100 folds' magnification. "Pa" is Pascal, showing the vacuum pressure of thesample chamber. Usually the SEM examines dry material, while a wet subject was examined by SEM, when the vacuum pressure was 270 Pascal, the humidity was saturated in the chamber and the temperature is $-10^{\circ} \mathrm{C}$. Somewet samples were studied by the SEM, e.g. expelled placental villi [4].

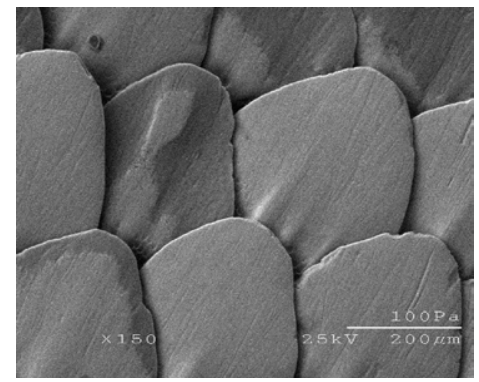

Figure 1: Scanning electron microscopic (SEM) finding of a shirk skin scales obtained at the abdomen of shirk fish showing smooth surfaces.

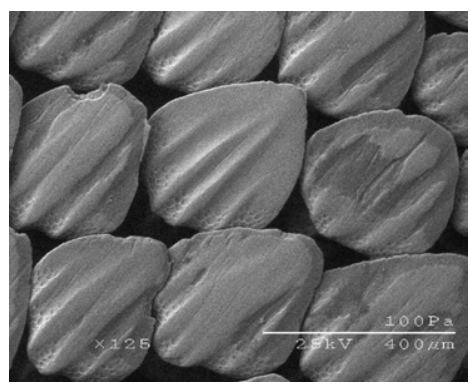

Figure 2: SEM finding of shirk fish skin scales obtained at the back of shirk fish showing uneven surfaces, which is utilized to polish various materials.

\section{Results}

\section{Shirk-skin scales}

Scales covering the skin of shirk fish, which is swimming in the close sea to our city, is examined by SEM. (Figure 1) shows the smooth surface of the scale of shirk skin obtained at the shirk abdomen (Figure 2), which was covered by strives were scales on the back of shirk (Figures 1 and 2).

Numerous protrusions develop at the sole of a wall lizard, which may make it possible to walk on the perpendicular smooth wall (Figures 3-5).

The beautiful structure shown on the scanning electron microscopic images on the leaf of a deutzia.

Beautiful star-like structures develop at the surface of deutzia leaves (Figures 6 and 7).

\section{Gourd-shape creatures on the leaf of gourd (Figure 8).}

\section{Discussion and Conclusion}

Micro-structures of the surface observed by scanning electron microscope were studied in the shirk skin scales, gecko extremity sole, leaves of some plants, and the placental villi of PIH in human subjects.

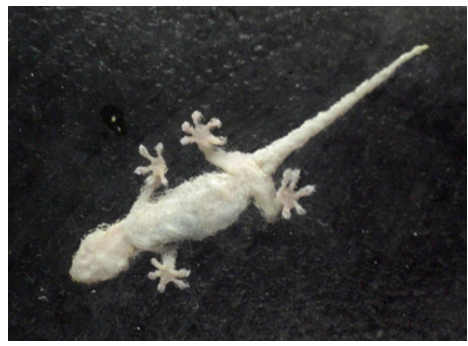

Figure 3: A gecko (wall lizard), which was walking on the window glass with adhesive soles.

${ }^{*}$ Corresponding author: Kazuo Maeda, Department of Obstetrics and Gynecology (Emeritus), Tottori University Medical School, Yonago, Japan, Tel: 81-859-22-6856; E-mail: maedak@moha.ocn.ne.jp

Received July 29, 2014; Accepted September 27, 2014; Published September 30, 2014

Citation: Tanaka K, Maeda K (2014) Scanning Electron Microscopy. J Health Med Informat 5: 167. doi:10.4172/2157-7420.1000167

Copyright: ( 2014 Tanaka K, et al. This is an open-access article distributed under the terms of the Creative Commons Attribution License, which permits unrestricted use, distribution, and reproduction in any medium, provided the original author and source are credited. 


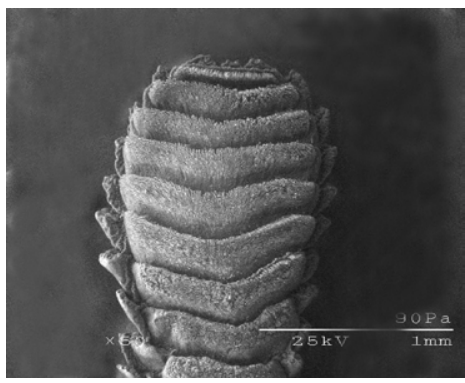

Figure 4: The SEM finding of the gecko extremity soles with mild augmentation.

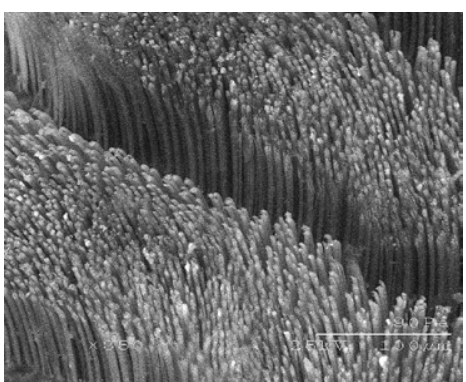

Figure 5: The SEM finding of gecko sole observed with high augmentation, where numerous protruded structures are noted to adhere the smooth surface of glass window.

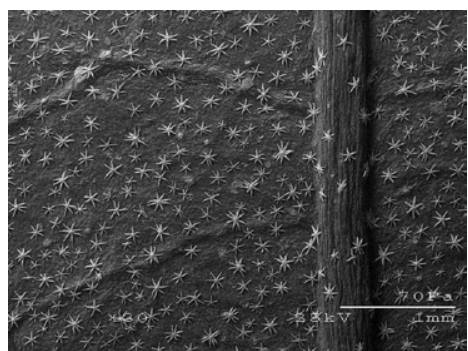

Figure 6: Nice structures found by the SEM in a deutzia leave, with mild augmentation.

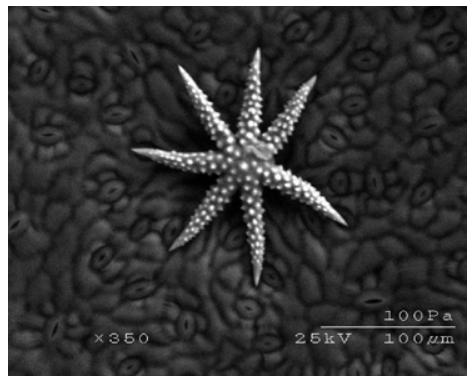

Figure 7: Single structure of deutzia leaf with high SEM augmentation.

Dry shark skin is used to polish various materials due to its unique surface structure shown in the scanning microscopic images.

Recently it was reported that an adhesive tape was investigated by the application of the structure of gecko sole shown in the scanning electron microscopic images.

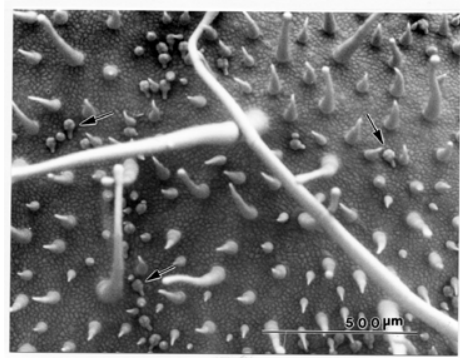

Figure 8: Strange structures found by SEM study on the gourd leaves.

( A)

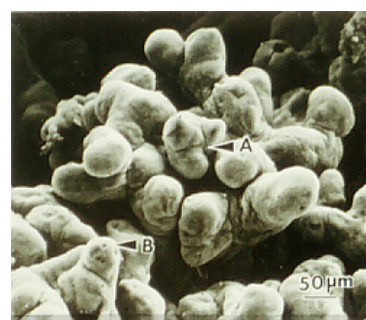

Figure 9: SEM findings of expelled placental villi in normal pregnancy (A) and severe pregnancy induced hypertension $(\mathrm{PIH})$. $(\mathrm{B})$, where the villi were thin and hypoplastic in the PIH placenta comparing to the placenta of normal pregnancy (Courtesy of Dr, O. Tsubaki of Tottori University [4]).

Human placenta were studied in this article with big interests, where a reason of fetal growth restriction was suspected by the low placental transfer of nourishment from maternal blood to the fetus by the thin and small villi of pregnancy-induced hypetension $(\mathrm{PIH}$, preeclampsis) placenta. Not only perinatal dysfunction in the PIH but also various health and medical problems will be clarified in the PIH using the SEM study [4]. The 3-dimensinal stereoscopic view of human placental villi studied in preeclampsia placenta, as shown in (Figure 9), will clarify possible cause of fetal abnormalities in the PIH by the atrophic placental villi. The structural finding obtained by the SEM is sometimes advantageous to 2-dimensional transparent microscopic view in scientific studies.

\section{References}

1. Tanaka K (1989) A Challenge to Ultramicro-world magnified for 800,000 Times Iwanami Publishing. Tokyo.

2. Pawley, Schatten (2008) Biologial Low-Voltage Scanning Electron Microscopy, Springer Newyork.

3. Schatten (2013) Scanning Electron Microscopy for the Life Sciences (Advances in Microscopy and Microanalysis), Cambridge University, London.

4. Tsubaki O (1979) Scanning electron microscopic studies on the placental villi in toxemia of pregnancy. Acta Obstet Gynecol Jpn: 31: 537. 